\title{
Hacia una construcción de subjetividades: a propósito del poder psiquiátrico de Michel Foucault en Cachaza de Virgilio A. Mora Rodríguez
}

\author{
Annamaria Rimolo BariattI \\ Escuela de Lenguas Modernas \\ Universidad de Costa Rica
}

\begin{abstract}
Resumen
El objetivo del presente artículo es presentar un análisis foucaultiano sobre el poder psiquiátrico (mediante tecnologías de poder y estrategias de disciplina y normalización) en la novela costarricense Cachaza de Virgilio A. Mora Rodríguez ${ }^{1}$. Se espera construir las diferentes subjetividades encontradas en la novela: la manera cómo el personaje principal es sometido, a la vez que es testigo de su propia transformación subjetiva, deviniendo en un ser normalizado.
\end{abstract}

Palabras claves: construcción de subjetividades, Michel Foucault, poder psiquiátrico, Cachaza, Virgilio A. Mora Rodríguez, literatura costarricense

\begin{abstract}
This article pretends to present an analysis after Foucault about the psychiatric power (by power technologies and normalization as well as by discipline strategies) within the Costa Rican literary novel Cachaza of Virgilio A. Mora Rodríguez. It is expected to build different subjectivities found in this novel, the way how the main character is submitted being witness of his own subjective transformation, becoming a normalized being.
\end{abstract}

Key words: construction of subjectivities, Michel Foucault, psychiatric power, Cachaza, Virgilio A. Mora Rodríguez, Costa Rican literature 


\section{Introducción}

Para Michel Foucault, los temas de la subjetividad, el poder y el saber revisten gran importancia y se ocupa de ellos a lo largo de su obra. El objetivo del presente trabajo es analizar el poder psiquiátrico, siguiendo la vía foucaultiana de la subjetividad, el saber y el poder en la novela Cachaza del psiquiatra costarricense Virgilio A. Mora Rodríguez, al tomar como textos de base dos de las obras de Foucault Historia de la locura en la época clási$c a$, volúmenes I y II (1992) y El poder psiquiátrico (2005).

Asimismo, Michel Foucault agrupó dos tipos de archivos, ambos del siglo XIX -Yo, Pierre Riviere, habiendo degollado a mi madre, a mi hermana $y$ a mi hermano (1973) y Herculine Barbin, llamada Alexina B. (1978)-, cuyas narraciones diversas una de la otra, concatenan la construcción de subjetividades, que por medio de una técnica de poder -la disciplina- se transforman en individuos disciplinados, presentándose así la construcción de subjetividades por el poder disciplinario a través de discursos médicos, judiciales, educativos y testimoniales. De la misma manera, en la obra Cachaza, el personaje central es sometido, a la vez que es testigo de su propia transformación subjetiva, deviniendo en un ser normalizado.

En el primer apartado de este artículo tenemos la narración de Cachaza y en el segundo, el tema de la locura en Michel Foucault en dos de sus obras. En el tercer y último apartado analizaremos la sociedad disciplinaria con sus temas adjuntos del poder disciplinario y el poder psiquiátrico. En todas las secciones anteriores se pretende analizar la construcción de subjetividades en Cachaza a la luz de las tecnologías del poder psiquiátrico. Finalmente, se trazan algunas conclusiones.

Como se mencionó anteriormente, la subjetividad es un aspecto recurrente en la obra de Foucault, pese a su constante cambio de enfoques, instrumentos de análisis y temas de estudio. En su trabajo, Foucault trata de responder a la pregunta ¿cómo se constituyen los individuos en sujetos en nuestra modernidad? Para poder responder al tema de cómo los sujetos llegan a ser lo que son, Foucault (1992) propone utilizar dos estrategias analíticas: la arqueología y la genealogía, esta última posterior.

\section{Estrategias analíticas: la arqueo- logía y la genealogía}

Ambas estrategias foucaultianas pueden ayudar en la construcción de subjetividades. El eje que nos ocupa en este ensayo es el relacionado con el poder. La estrategia analítica de la arqueología se desarrolla desde finales de la década de 1950 hasta finales de la década de 1960. Mediante un análisis histórico de diferentes saberes como la medicina y la psiquiatría, Foucault busca en los diversos discursos cómo pudieron formarse las subjetividades. La estrategia analítica de la genealogía consiste en analizar la historia de las subjetividades modernas mediante tecnologías de poder y estrategias de disciplina $y$ normalización que toman en cuenta el cuerpo humano, el cuerpo sometido, vigilado, marcado, utilizado, castigado, disciplinado, en fin, normalizado. Por ello, al analizar la construcción de subjetividades a la luz del poder psiquiátrico en Cachaza, hay que tomar en 
cuenta las relaciones de poder, el campo del saber psiquiátrico y los procesos de normalización en psiquiatría.

\section{Diégesis en Cachaza}

La novela Cachaza -del psiquiatra costarricense Virgilio Mora Rodríguezfue originalmente publicada en $1977 \mathrm{y}$ reeditada treinta y dos años después por la Editorial UNED en 2009. En aquel tiempo, fue considerada narrativa innovadora, pues incorporó tanto un lenguaje pachuco ${ }^{2}$ como temas censurados en el discurso literario tradicional costarricense (la locura, la vida sexual, lo indecente -lo que no se dice ni se escribe públicamente). La novela es la historia -en los alrededores del Mercado Central, la Penitenciaría y el Asilo Chapuí ${ }^{3}$ - de un personaje, Cachaza, contada por él mismo en un monólogo.

El personaje principal Cachaza, quien inmediatamente después de presenciar de niño la muerte de su padre a manos de los insurgentes del levantamiento de $1948^{4}$, se convierte en un pordiosero del Mercado Central de San José, en un preso en la Penitenciaría y finalmente, en un loco en el Asilo Chapuí. Allí se hace pasar por mudo y demuestra una gran lucidez al describir todos los detalles del diario acontecer en el asilo y en las vidas de los pacientes. La novela es "una fuerte denuncia sobre la situación que se daba en el Asilo Chapuí, encarnada en personajes verosímiles y estremecedoramente humanos" (Fonseca y Rivera, 1979, p.8).

Dentro del asilo, Cachaza finge ser mudo para que no le den de alta pues no tiene dónde ir y es quien mejor sabe de lo que está ocurriendo en el hospital. Se convierte en un ayudante indispensable: asiste al doctor Montuno con la máquina de electrochoques, lava el carro a los médicos, hace mandados, borra la pizarra los días de presentaciones de casos y ayuda a bañarse a los pacientes que no pueden hacerlo por sí mismos.

Tal situación del personaje principal le permite a Mora Rodríguez contar con un punto de vista privilegiado -tal y como lo hizo Foucault al iniciar su estudio sobre la locura en los psiquiátricos-. Desde allí puede inmiscuirse en la historia y la vida cotidiana del hospital y mostrar la cruel situación de abandono en que viven los enfermos y la irresponsabilidad del personal tanto médico como auxiliar. Nadie cumple con las ocho horas laborales, les interesa más un partido de fútbol que la vida de un paciente, toman licor en el asilo y duermen las borracheras ahí mismo, a vista y paciencia “...del doctor Guvila, el director, un hombre que cuando está en el Asilo no sale, se encierra en su oficina y que en los últimos cinco años de su reinado en este lugar, no ha visto un solo enfermo..." (Mora Rodríguez, 2009 , p.9). En cuanto a las enfermeras, se la pasan teniendo sexo con los doctores o bien recetando a los pacientes.

Mediante el monólogo de Cachaza, se describe la vida de los enfermos mentales, sus necesidades reales -viven en sus propios excrementos y comen lo mismo todos los días-, tanto en los niveles afectivo como alimentario que día tras día, al ser llevados a los electrochoques y al ser medicados, van perdiendo su identidad y se convierten en sujetos disciplinados. Estos

... pacientes que los doctores llaman crónicos incurables, hombres, mujeres, viejos, jóvenes sin pasado, sin futuro, hombres que viven lo que les queda de 
vida en segundos, para los que no existe ayer ni mañana ni tarde ni temprano, hombres, mujeres, viejos, jóvenes, cuyos días y noches se les volvieron instantes en los que viven aprisionados, olvidados de todos, nada existe en sus vidas..." (Mora Rodríguez, 2009, p.126)

Así, se van conociendo tanto los diversos pabellones del hospital (aislamiento, prestinari -sector del hospital donde pasean-, la sala de electrochoques) como las calamidades, sufrimientos y abandono de los enfermos. También a través del relato se hace un paralelismo entre el Chapuí (asilo) y la Penitenciaría (prisión), no se encuentran casi diferencias y cuando las hay, sale ventajosa la Penitenciaría.

La historia termina cuando el 24 de diciembre, en la fiesta de Noche Buena, Cachaza ve partir al Viejito - un paciente que es su mejor amigo, a quien vistió con una corbata vieja y peinórumbo al Hospital San Juan de Dios. Sorpresivamente un médico descubre, por primera vez y después de muchos meses de estar ahí, que el Viejito tenía un tumor cerebral. Cachaza no resiste la separación y grita desesperadamente que no se lo lleven. Tal es su desesperación, que gesticula, grita, se enfurece y se pone agresivo. Finalmente se lo llevan y, poco a poco, los fuertes electrochoques, el efecto de las múltiples píldoras, la poca comida y la mala atención, van convirtiéndolo en

... un ser humano muerto en vida cuya paz de día y de noche, rayaba en los límites de la muerte. Lo privaron del derecho de existir; de ver; oler, oír; sentir... lo redujeron, con la excusa de curarlo, en la caricatura grotesca de un ser humano perdido y derrotado, olvidado y encerrado con cerradura especial en una de las celdas, pocilgas del Servicio de Aislamiento, mientras todo el mundo en la ciudad, todos los doctores y las enfermeras del Hospital, se deseaban a gritos, que tengas un año nuevo muy feliz... (Mora Rodríguez, 2009, p.141)

\section{Sobre el tema de la locura en Michel Foucault}

Mediante las estrategias analíticas de la arqueología y de la genealogía, Michel Foucault estudia la locura. Dos de sus libros Historia de la locura en la época clásica, volúmenes I y II (1992) y El poder psiquiátrico (2005) son utilizados en este ensayo para el análisis de cómo el personaje principal, Cachaza, de la novela del mismo nombre, Mora Rodríguez construye su subjetividad.

\section{Sobre Historia de la locura en la época clásica, volúmenes I y II}

Michel Foucault publicó esta obra en 1964 y constituye uno de sus primeros trabajos sobre el tema de la locura, antecedida por Enfermedad Mental y Personalidad (1954). Estos dos volúmenes (sexta reimpresión de 1992) están estructurados en tres partes. En el prólogo, Foucault hace referencia a la relación de poder que establece el autor con el lector pues el poder del autor sobre su obra es el “...primer acto por el cual comienza a establecerse la monarquía del autor, declaración de tiranía: mi intención debe ser vuestro precepto, plegaréis vuestra lectura, vuestros análisis, vuestras críticas a lo que yo he querido hacer" (Foucault, 1992, p.8). 
Este poder está dado por la proclama que hace Foucault de ser el monarca de sus ideas. Ya desde aquí se ve la relación del saber, el poder y el sujeto.

La primera parte está compuesta de cinco capítulos ("Stultifera Navis", El gran encierro, El mundo correccional, Experiencias de la locura y Los insensatos). Su primer capítulo está basado en el poema de Sebastián Brant Das Narrenschiff (1492), donde se relata el viaje de varios personajes con diferentes vicios en una nave y hace referencia a los motivos de la exclusión social de los que sufrían lepra, enfermedades venéreas y locura durante la Edad Media.

De todos estos navíos novelescos o satíricos, el Narrenschiff es el único que ha tenido existencia real, ya que sí existieron estos barcos, que transportaban de una ciudad a otra sus cargamentos insensatos. Los locos de entonces vivían ordinariamente una existencia errante. (Foucault, 1992, p.21)

Por otro lado, Foucault señala que la locura fascina porque es deseo y saber: un saber cerrado y difícil de alcanzar:

¿Qué anuncia el saber de los locos? Puesto que es el saber prohibido, sin duda predice a la vez el reino de Satán y el fin del mundo...La victoria no es ni de Dios ni del Diablo; es de la Locura. (1992, pp. 40-41).

Como vemos, dentro del imaginario europeo la locura estaba asociada al agua. Sin embargo, ya en el Renacimiento se pasa de la nave al hospital de locos, del embarco al encierro. En el segundo capítulo El gran encierro, Foucault hace referencia a las reformas sanitarias desde la proclama de
1656 con el decreto de la fundación del Hospital General en París cuya función era regular administrativamente la locura. Se establece el poder de los directores nombrados de por vida, quienes poseen

... todo poder de autoridad, de dirección, de administración de comercio, de policía, de jurisdicción de corrección, sanción sobre todos los pobres de Paris, tanto dentro como fuera del Hospital General. (1992, p.81)

En Cachaza vemos esta investidura autoritaria en el director del Asilo Chapuí Dr. Guvila y en su próximo sucesor el Dr. Gallant, quien

... no hace mucho regresó de los Estados Unidos en donde dice que se hizo psiquiatra en la Clínica Mayo de donde trajo un montón de recibos, cartas, constancias, fotos que a todo el mundo enseña, dicen que ya muy pronto va a reemplazar al doctor Guvila que es muy "güevón" y que habla solo "paja" y que para todo cita a Fromm. (Mora Rodríguez, 1992, p.10).

En el tercer capítulo Foucault hace una crítica a la medicalización de la locura, a la política del internado y a la sensibilidad social en las que se ha formado la conciencia médica de la locura: “...y hasta qué punto le sigue estando atada, puesto que es esta sensibilidad la que sirve de elemento regulador cuando se trata de decidir entre un internamiento o una liberación" (Foucault, 1992, p.127).

Lo mismo encontramos en Cachaza:

... cuando tengan un paciente en las condiciones del señor de que nos ocupamos esta mañana, por favor no me lo 
manden a mi oficina, no tengo que explicarles el porqué, todo el mundo sabe que este paciente que hoy se discute, se defecó y se orinó apenas me vio, cuando tengan un caso como éste, mándenlo a Observación y yo se los veo ahí... (Mora Rodríguez, 2009, pp.20-21).

En el cuarto y quinto capítulos de esta primera parte (Experiencias de la locura y Los insensatos), Foucault nos habla de dos arqueologías: la del silencio para referirse a todo un corpus de referencias que se manejan para silenciar la locura durante la época clásica y la arqueología médica dedicada a decidir quién está loco y quién no y cuáles son las medidas para reprimir la locura. En Cachaza se ven acciones dirigidas a curar aunque más parezcan tortura.

Se le tiran encima dos hombres musculosos expertos en "llaves" y tres mujeres, todos "auxiliares de enfermería". De los hombres, el más grande, un indiazo, las espaldas y los brazos llenos de músculos como pelotas, se ríe de sus esfuerzos inútiles y ya dominada le da una gran nalgada al tiempo que el otro comenta muerto de la risa, qué hijueputa loca más loca...Grita, se revuelca aunque ya la han amarrado al catre y el compañero del indiazo vuelve a repetir, qué hijueputa loca más loca, ahora hablando con voz gangosa pues se tapa la nariz para evitar la hediondez a mier$\mathrm{da}$, a orines. Una rata tan grande como un gato, corre, le pasa por entre las piernas a la enfermera que grita como si arremedara a la loca al tiempo que clava la aguja de la nueva inyección en el colchón viejo y hediondo, ¿cambiamos la aguja doctor?, ¿para qué?, dale otro chuzazo a ver si se calma y anotala en la lista del doctor Montuno para que le den una serie de maquinazos. (Mora Rodríguez, 2009, p.2)

La segunda parte de Historia de la locura en la época clásica, volúmenes I y II consta de cuatro capítulos donde Foucault (1992: 258-266) presenta las diferentes formas de conciencia: la conciencia crítica de la locura (lo razonado, lo reflexionado, lo moralmente sabio) que no define ni denuncia; la conciencia práctica de la locura que está relacionada con la realidad concreta; la conciencia enunciadora de la locura que da la posibilidad de decir en lo inmediato sin desviación por el saber; y la conciencia analítica de la locura (desplegada de sus formas, de sus fenómenos y de sus modos de aparición). Mientras en la época clásica se privilegiaba la conciencia crítica y la conciencia práctica, en los siglos XIX y XX, es la conciencia analítica. La tercera parte consta de cinco capítulos y se señalan los elementos de la socialización y la construcción subjetiva de la individualidad pero mediada por frenos sociales como la libertad, la religión, el tiempo, la civilización y la sensibilización.

\section{Sobre El poder psiquiátrico}

El libro El poder psiquiátrico (2005) conforma los cursos impartidos por Michel Foucault a finales de 1973 y principios de 1974 sobre el poder psiquiátrico, continuando así con el proyecto en su obra Historia de la locura en la época clásica, volúmenes I y II sobre una historia de las ciencias humanas. En ella se refiere a la línea divisoria que separaba en nuestras sociedades al loco del no loco. La obra termina con la medicalización de la locura a principios del siglo XIX. En cambio, en este 
curso de 1973-74 Foucault da un giro y propone una genealogía de los movimientos antipsiquiátricos que tanto han marcado la década de 1960. Más al respecto se analizará en el siguiente apartado de este artículo.

\section{La sociedad disciplinaria}

En Foucault, el poder psiquiátrico está enmarcado dentro del tema de la sociedad disciplinaria -que se construye mediante una red de aparatos o 'dispositifs' que producen y regulan costumbres y hábitos- y, por lo tanto, se relaciona con el poder disciplinario y la anormalidad. En efecto, el poder disciplinario estructura los parámetros del pensamiento, sancionando y prescribiendo los comportamientos normales y/o desviados.

\section{El poder disciplinario}

En su libro Vigilar y castigar (publicado por primera vez en 1975), se recogen las principales tesis sobre el poder disciplinario. Recordemos también que al agrupar dos dossiers del siglo XIX (Yo, Pierre Riviere, habiendo degollado a mi madre, a mi hermana y a mi hermano, de 1973 y Herculine Barbin, llamada Alexina B., de 1978), se mostró que por medio del poder disciplinario los individuos se transforman en sujetos disciplinados y sus comportamientos son normalizados mediante tecnologías disciplinarias de poder. Ejemplos de estas tecnologías lo son la prisión y la disciplina carcelaria, el manicomio y la medicalización en el asilo.

Según Foucault, el poder disciplinario logra ajustar la singularidad corporal a la función sujeto, es decir, aplica una función sujeto o de sujeción sobre tiempos, fuerzas, cuerpos, gestos. El poder disciplinario fabrica 'cuerpos sujetos', fija la función sujeto al cuerpo. En Cachaza, se fabrican individuos disciplinados como el delincuente cuando el personaje principal entra en la Penitenciaría. Al respecto, Cachaza dice sobre sí mismo:

... yo era bueno antes de que me encarcelaran, en la Peni me eché a perder con el Mitajuan, el Treinta Años y el Frijolillo, ellos me enseñaron a fumar marihuana, yo no les quería aceptar pero como los veía tan alegres después de que fumaban, me animé, les pedí que me vendieran un cigarrillo, vale un colón, solo tengo seis reales, está bien. Mira cómo se fuma, chúpate el humo con ganas, hasta adentro, no lo sueltes hasta que sientas que te vas a reventar, ¿estás seguro de que esto no me va a volver loco?... (Mora Rodríguez, 2009, p.51).

El poder disciplinario presenta varias características. En primer lugar, es un poder anónimo repartido entre varias personas dentro del sistema como médicos, juristas y vigilantes. No está concentrado pues no descansa en una sola persona como el poder soberano, sino que busca recaer sobre un cuerpo, para convertirlo en alguien dócil y sumiso. Al recibir Cachaza los electrochoques para 'disciplinarlo' y 'normalizarlo', se hace mediante un cuerpo de médicos y auxiliares.

A las ocho de la mañana dos auxiliares abren la puerta de su pocilga y colgando de las piernas y de los brazos, como si solo fuera un paquete de carne y huesos envuelto en trapos viejos, se lo llevan al 
cuarto de electrochoques. En la oficina del Servicio de Aislamiento, el doctor Montuno, como siempre, habla a gritos, espero que ya me tengan a todos los pacientes listos, ustedes saben que no me gusta dar electrochoques en seco... La enfermera, dos auxiliares, el doctor Montuno y el doctor Restrepo, esta mañana de lentes oscuros y mascando chicle, se dirigen al cuarto de tratamientos. Por entre dos hileras de catres pegados el uno contra el otro, la enfermera, los dos médicos y el auxiliar que carga la máquina, se deslizan de medio lado hacia el fondo, mientras el otro auxiliar permanece parado al pie de la puerta, listo por lo que pueda suceder... (Mora Rodríguez, 2009, pp.144-145).

En segundo lugar, el poder disciplinario logra tocar, modificar y dirigir cuerpos y almas mediante la incorporación de métodos disciplinarios en la vida cotidiana. Cachaza recuerda cuando era recluido en un recinto de aislamiento total:

... soñé que estaba de nuevo en La Tumba, viejito, una celda en la que me encerraron muchas veces cuando yo era un reo por lo que me pasó en el Mercado Central, la de horrores que pasé en la Peni, las hambres, tantas que terminé aprendiendo a comer poquitos, a pasármela por semanas a pan y agua, me acostumbré a estarme quieto, sin moverme, sin dormir, encerrado en un hueco oscuro, lleno de murciélagos que a veces me atacaban... (Mora Rodríguez, 2009, p.51).

Una tercera característica del poder disciplinario es que ocupa el cuerpo y el tiempo del individuo. Es un procedimiento de control constante, en que el individuo queda a merced de la observación constante. Encontramos en el poder disciplinario ejercicios calculados y repetidos. Finalmente, una cuarta característica es lo que Foucault llama 'escritura', id est, anotar y registrar todo lo que el individuo hace y dice, todo lo que le ocurre. En Cachaza encontramos este control constante, continuo y perpetuo en los tratamientos aplicados (electrochoques) y su debida anotación en los expedientes de los pacientes.

Esta idea del poder disciplinario, que garantiza la visibilidad del individuo, continúa en la obra foucaltiana $E l$ poder psiquiátrico (1973-74) y se amplía en Los anormales (1974-75).

\section{El poder normalizador}

Relacionado con el poder disciplinario está el poder normalizador. La anormalidad es un asunto tratado ampliamente en el curso 1974-75 sobre los anormales. Los médicos, juristas y educadores son investidos de conocimiento científico y con intención normalizadora. En Cachaza encontramos intentos de marcar la degradación que hay desde lo normal hasta lo anormal aplicados en el campo de la sexualidad:

... sí, doctor, esa mujer está completamente psicótica, para mí que se trata de un episodio agudo de pánico homosexual, parece tener una psicopatología en la que la esfera sexual juega el papel más importante, el componente homosexual es palmario... (Mora Rodríguez, 2009, p.17).

En el discurso del peritaje psiquiátrico encontramos dos tipos de estrategias. 
Una de ellas es la duplicación. Los trabajadores de la salud duplican el delito con una serie de componentes como maneras de ser que presentan la causa, el origen, la motivación, el punto de partida del delito y como marcas lingüísticas se encuentran expresiones como 'inmadurez psicológica', 'personalidad poco estructurada', 'desequilibrio afectivo', 'serias perturbaciones emocionales' y 'juego perverso'. Estas marcas repiten el delito para inscribirlo como rasgo individual. Por ejemplo, en la novela de Mora Rodríguez a la Loca Prado se le repetía constantemente "que loca más loca". Otra estrategia del peritaje psiquiátrico es el continuum de la vida peligrosa donde el delincuente demuestra signos de anormalidad desde su infancia. Esto muestra cómo el individuo se parecía ya a su crimen antes de haberlo cometido y se da una reconstrucción anticipatoria del acto criminal. Esto se ve en Cachaza quien desde pequeño sufre el gatillo desencadenante que lo lleva a la construcción de su propia subjetividad. Aunque Cachaza no es un 'monstruo humano', es traumatizado a los cuatro años cuando presencia la muerte de su padre:

... esos tipos lo mataron... aún los veo tirándoseles encima, solo sus puños... las culatas de los máuseres... le destrozaron la cara, le arrancaron la nariz, le tumbaron todos los dientes... un solo culatazo y le destrozaron la cara... la cara de mi padre que aquí terminaron de destrozarme a maquinazos convirtiéndola en nada... mi padre... se les tiró encima, Lario, míralo... se les volvió a ir encima después de que le habían arrancado la nariz... ¿oíste ese balazo?... le destrozaron la cabeza de un tiro... ¿oyes ese otro disparo?... un balazo en el pecho, le destrozaron el corazón... el mío se paró, yo sentí que me moría... lo dejaron tirado en un hueco que él mismo hizo a pico y pala, solo... no quiso que le ayudara, yo que sabía que ahí mismo esos hombres lo pensaban enterrar... no lo enterraron, lo tiraron en el hueco como si fuera un perro... (Mora Rodríguez, 2009, pp.136-137).

Como vemos, el poder disciplinario está muy relacionado con el poder psiquiátrico como se aprecia a continuación.

\section{El discurso psiquiátrico}

Como dijimos anteriormente, la Historia de la locura en la época clásica, volúmenes I y II efectuaba la línea divisoria que separa al loco del no loco y finaliza con la medicalización de la locura a principios del siglo XIX. En su obra El poder psiquiátrico, Michel Foucault discute los conceptos que diez años antes utilizó:

Por lo tanto, en lugar de hablar de violencia, me gustaría más hablar de microfísica de poder; en vez de hablar de institución, me gustaría más tratar de ver cuáles son las tácticas puestas en acción en esas prácticas que se enfrentan; en lugar de hablar de modelo familiar o de "aparato del Estado", querría intentar ver la estrategia de esas relaciones de poder y esos enfrentamientos que se despliegan en la práctica psiquiátrica. Ustedes me dirán que está muy bien haber sustituido violencia por microfísica del poder, institución por táctica, modelo familiar por estrategia, pero ¿acaso avancé? He evitado términos que permitían introducir el vocabulario psicosociológico en todos estos análisis, y ahora estoy frente a un vocabulario pseudomilitar que no 
debe gozar de mucha mejor fama. Pero vamos a tratar de ver qué se puede hacer con eso. (Foucault, 2005, p.34).

Desde ese punto de vista, el tema del poder psiquiátrico prosigue el proyecto de una historia de las "ciencias" humanas. El curso concluye a finales del siglo XIX en el momento en el que se produce la doble "despsiquiatrización" de la locura, dispersa entre el neurólogo y el psicoanalista. De este modo, en El Poder psiquiátrico, Foucault propone una genealogía de los movimientos antipsiquiátricos que tanto han marcado la década de 1960.

El curso consta de trece clases: cinco de ellas se localizan en el asilo y desarrollan aspectos del poder disciplinario (el orden y la disciplina, el tema de la fuerza) y las últimas ocho lecciones se concentran en el tema del poder psiquiátrico, específicamente las marcas del saber psiquiátrico y los debates médicos acerca de síntomas y curaciones. Foucault inicia citando a autoridades francesas de la primera mitad del siglo XIX como Pinel y Fodéré, quienes señalan que el orden y la disciplina son necesarias para construir el saber médico de manera objetiva y para curar permanentemente; por ejemplo, en la descripción que Fodéré hace del asilo ideal,

... en su interior, desde luego, reina el orden, reina la ley, reina el poder. Dentro de ese decorado, en ese castillo protegido por una ambientación romántica y alpina, en ese castillo sólo accesible mediante el uso de complicadas máquinas, y cuyo aspecto mismo debe sorprender al común de los hombres, impera ante todo y simplemente un orden, en el sencillo sentido de una regulación perpetua y permanente de los tiempos, las actividades, los gestos; un orden que rodea los cuerpos, los penetra, los trabaja... (Foucault, 2005, p.16)

En Cachaza, observamos que el poder y el orden reinan mediante la aplicación continua de los electrochoques y la medicación a los pacientes del Asilo Chapuí dirigida a sojuzgar su voluntad y convertirlos en sujetos disciplinados. Ya sea controlando o curando, los médicos son titulares de esta fuerza. Pero no están solos: tienen ayudantes y vigilantes. En el Asilo Chapuí los auxiliares y las enfermeras ejercen estos roles.

Otra característica del poder psiquiátrico es el tema de la fuerza. A comienzos del siglo XIX se consideraba que el loco desencadena cierta fuerza la del loco furioso, la de las pasiones, la del instinto- que debe ser enfrentada. Esta táctica del asilo, la de reconocer la fuerza del paciente para someterlo, subyugarlo y domesticarlo es de gran importancia para el paciente pues sin ella -sin pasar por el poder psiquiátrico- no podrá ser curado. Este es el quid de la cuestión: la sumisión como curación. Foucault en su segunda clase relata el caso -sacado de un texto de Pinel- del rey de Inglaterra Jorge III, quien se somete al médico para curarse de su locura:

... el alienado, alejado de su familia y de todo lo que lo rodea, queda confinado en un palacio aislado y se lo encierra solo en una recámara cuyos cristales y muros se cubren de colchones para impedir que se hiera. Quien dirige el tratamiento le informa que ya no es soberano y le advierte que en lo sucesivo debe mostrarse dócil y sumiso. Dos de sus antiguos pajes, de una estatura hercúlea, quedan a cargo de 
atender sus necesidades y prestarle todos los servicios que su estado exige, pero también de convencerlo de que se encuentra bajo su entera dependencia y de que de allí en más debe obedecerlos. Guardan con él un tranquilo silencio, pero en cuanta oportunidad se les presenta le hacen sentir la superioridad de su fuerza. (Foucault, 2005, p.36)

No está de más decir que luego de este tratamiento, el monarca encuentra su curación. No sin antes despojarse de su poder soberano y reemplazarlo por

... un poder que podríamos calificar de disciplina y cuyo efecto no consiste en absoluto en consagrar el poder de alguien, concentrar el poder en un individuo visible y con nombre, sino en recaer únicamente en su blanco, sobre el cuerpo y la persona misma del rey descoronado, al que ese nuevo poder debe hacer ‘dócil y sumiso'. (Foucault, 2005, p.39)

Como observamos, el discurso psiquiátrico configura su poder mediante el poder disciplinario. Como Foucault lo señala:

La hipótesis que quiero proponer es que en nuestra sociedad existe algo que podríamos llamar poder disciplinario. Por ello no entiendo otra cosa que cierta forma terminal, capilar del poder, un último relevo, una modalidad mediante la cual el poder político y los poderes en general logran, en última instancia, tocar los cuerpos, aferrarse a ellos, tomar en cuenta los gestos, los comportamientos, los hábitos, las palabras... creo que el poder disciplinario es una modalidad determinada, muy específica de nuestra sociedad, de lo que podríamos denomi- nar contacto sináptico cuerpo-poder. (Foucault, 2005, pp. 59-60).

Por lo tanto, el poder psiquiátrico se comprende en el marco del poder disciplinario pues la locura solo puede curarse después de haber pasado por el poder psiquiátrico. En los asilos sin el orden y la disciplina, no es posible la curación. Para ello, el médico debe ser titular y marcar en el cuerpo de quien ejerce el poder. Es ayudado por los vigilantes y los ayudantes, quienes ejercen un determinado sitio y una función -al igual que el médico- para el ejercicio del poder. Sin embargo, el poder disciplinario tiene sus límites pues hay ciertos elementos que escapan de su vigilancia -desertores, delincuentes, débiles mentales, monstruos morales, incorregibles-. Por ello, el tema de los anormales y del poder de normalización está también relacionado con el discurso psiquiátrico.

\section{Algunas conclusiones}

En Cachaza vemos la construcción de subjetividades, el tema central de Foucault de cómo los sujetos llegan a ser lo que son: de cómo Cachaza llega a ser lo que es. Y alrededor del tema del sujeto están los temas del saber y del poder. Vivimos en sociedades disciplinarias con aparatos o 'dispositifs' que hacen una variedad de cosas: producen y regulan costumbres, hábitos y prácticas; aseguran la obediencia a sus reglas; ponen en marcha mecanismos de inclusión y/o exclusión; poseen instituciones disciplinarias como el asilo; nos gobiernan; estructuran nuestros pensamientos; al tiempo que sancionan nuestros comportamientos normales 
y/o anormales. Este poder disciplinario lleva a los que no entran en el apartado de 'los incluidos' a un fin mortal. Al igual que el Pierre Riviere y la Alexina de Foucault, Cachaza tiene su mismo fin: el suicidio, en el sentido de que al final se deja vencer por el asilo. Cuando entra en crisis debido a la muerte de su amigo El Viejito, se le aplica todo el poder disciplinario del discurso psiquiátrico:

\begin{abstract}
Sigamos con el Cachaza, Restrepo. Míralo cómo duerme. Te garantizo que después de esta serie va a quedar irreconocible. Señorita, ¿qué me dice de este paciente?, ¿algún problema? Ninguno doctor; el Cachaza ha estado durmiendo divino toda esta semana. La combinación de electrochoques y Haldol es magnífica. A ver señoritas; métale el trapo en la boca. Dale tú el electrochoque, Restrepo... (Mora Rodríguez, 2009, p.144).
\end{abstract}

En la novela Cachaza, la voz autorial de Virgilio Mora Rodríguez -psiquiatra costarricense- habla desde sus personajes y expone su punto de vista sobre la crudeza en el Asilo Chapuí sin caer en opiniones moralistas. La novela es en sí una clara denuncia social de una situación que aún reviste actualidad en nuestra sociedad costarricense, la que consideramos que se está deshumanizando cada vez más. Puede ser que el Asilo Chapuí de la novela ya no sea el mismo que denuncia Mora Rodríguez - que hayan cambiado sus condiciones sanitarias y los métodos aplicados a los pacientes-, pero los males sociales que nuestro sistema social, político y económico padece tienen la posibilidad de provocar en los individuos un estado depresivo o esquizofrénico -el mismo que sufrió precisa- mente Cachaza a lo largo de su vida. Esto significa que los hechos descritos en Cachaza aún los vemos en nuestra sociedad: corrupción, pérdida de valores y deshumanización.

Sin embargo, Foucault nos da una esperanza, una posibilidad de resistencia. Como dijimos anteriormente, el poder disciplinario tiene sus límites y algunos escapan de su vigilancia (son los desertores, los delincuentes, los débiles mentales, los monstruos, los incorregibles). Cada uno de ellos son su contraste, su punto ciego, su límite y solo tienen sentido frente a la norma disciplinaria. El sistema, sin ellos, no se sostendría, pues los necesita. Para Foucault es importante tomar en cuenta que el poder disciplinario tiene puntos donde no llega. Los anormales son ejemplos donde el poder disciplinario no tiene éxito.

Foucault nos sugiere que las sociedades disciplinarias fijan individuos dentro de instituciones, pero no logran dominarlos y consumirlos completamente, ni alcanzan por completo la conciencia y los cuerpos de los individuos, pues organizar la totalidad de sus actividades es imposible. Es decir, donde hay poder hay resistencia. En una sociedad disciplinaria no faltan 'esos individuos' como Cachaza que se escapan de la vigilancia continua y perpetua y logran denunciar la violencia institucional.

\section{Notas}

1 Este narrador y psiquiatra costarricense, radicado en Nueva York, utiliza varios nombres: V.A. Mora Rodríguez, V.A. Mora R. y Polo Mora. En la primera edición de Cachaza (Editorial Costa Rica, 1977) aparece como V. Mora R. 
2 En Costa Rica, jerga popular.

3 El Hospital Psiquiátrico Chapuí, en San José, Costa Rica

4 Guerra Civil o Revolución de 1948 en Costa Rica.

\section{Bibliografía}

Amoretti, María. "Cachaza: El relajo del carnaval y el diálogo de los contrarios". Káñina, VII (2), 21-22. San José: Universidad de Costa Rica.

Amoretti, María. Introducción al sociotexto: a propósito de Cachaza. San José: Editorial de la Universidad de Costa Rica, 1989.

Fonseca, Julieta y Marta Rivera. Cachaza: relato novedoso en la literatura costarricense. San José: Tesis, Universidad de Costa Rica, 1979.

Foucault, Michel. Historia de la locura en la época clásica. Volúmenes I y II. México: Fondo de Cultura Eco- nómica, 1992.

Foucault, Michel. Yo, Pierre Rivière, habiendo degollado a mi madre, a mi hermana y a mi hermano. Barcelona: Tusquets, 2001.

Foucault, Michel. El poder psiquiátrico. $1^{\mathrm{a}}$ ed., $2^{\mathrm{a}}$ reimpresión. Buenos Aires: Fondo de Cultura Económica, 2005.

Foucault, Michel. Herculine Barbin llamada Alexina B. Madrid: Talasa, 2007.

Foucault, Michel. Vigilar y castigar: nacimiento de la prisión. $2^{\mathrm{a}}$ ed. revisada. Argentina: Siglo XXI Editores, 2008.

Mora Rodríguez, Virgilio A. Cachaza. San José: Editorial Universidad Estatal a Distancia, 2009. 
